\title{
Self-Similar Characteristics of COVID-19 Patient arrival at Healthcare Centre - A Study Using Queuing Models
}

\author{
AM Girija ${ }^{a}$,*D.Mallikarjuna Reddy ${ }^{b}$, Pushpalatha Sarla ${ }^{c}$ \\ ${ }^{\mathrm{b}}$ Assistant Professor, Department of Mathematics, GITAM University, Hyderabad \\ ${ }^{a}$ Research Scholar, Department of Mathematics, GITAM University, Hyderabad \\ ${ }^{c}$ Assistant Professor, Dept. of Mathematics, SRITW, Warangal. \\ *mallik.reddyd@gmail.com
}

Article History: Received: 10 November 2020; Revised 12 January 2021 Accepted: 27 January 2021; Published online: 5 April 2021

\begin{abstract}
The entire world is spreading of coronavirus-COVID-19 has increased exponentially across the globe, and still, no vaccine is available for the treatment of patients. The crowd has grown tremendously in the hospitals where the facilities are minimal. The queue theory is applied for the Single-server system and its self-similarity existence in a queue used to identify the queue time, waiting time, and Hurst parameter by different patient arrivals methods Health care center in our local area located in Hosapete, Ballari district, Karnataka. Due to more arrivals to the health care center for the identification and confirmation of disease covid-19. This study paper presents a sequential queuing model for estimating infections' detection and identification in severe loading conditions. The goal is to offer a simplified probabilistic model to determine the general behavior to predict how long the treatment cycle will diagnose and classify people already tested and get negative or positive results. For this type of Method, there are some graphical representations of the various measurement criteria. The modelling results showed that the patient's waiting period in the course of inquiries, detections, detecting, or treating COVID-19 in the event of imbalances in the system as a whole rise following the logarithm rule.
\end{abstract}

Keywords: Queuing model, Self-Similarity, Hurst parameter, Probabilistic model, COVID-19

\section{Background}

\subsection{History of COVID-19(Coronavirus-2019)}

The COVID-19(coronavirus-2019) was formally declared as disease identified by the World Health Organization (WHO) on 11th February 2020. After the outbreak of COVID 19, over 200 countries and regions worldwide have been affected and faced significant challenges. A Probabilistic Model for the Assessment of Queuing Time of Coronavirus Disease (COVID-19) Patients using Queuing Model To date, more than 19 million cases worldwide have been confirmed, with total deaths over 700,000 (WHO, 2020). However, around 12 million patients have also recovered from the disease. The cases in India have also increased exponentially from June'2020. The daily infection rate is growing at a rapid pace. The 1.9 million cases were reported in India by the end of July 2020 , with fatalities of around 40,000. Most of the COVID-19 patients are found asymptomatic but may spread the virus to others. The asymptomatic infections lead to positive nuclear acid detection by reverse transcriptase-polymerase chain reaction (RT-PCR) samples. They are still not distinguished by a classic clinical symptom or sign or by apparent anomalies in medical transcription, like lung-computed tomography (C.T.) (Gao, W.J., Zheng, K., Ke, J., and Li, L.M., 2020). The sufficient identification of an infected individual and the elimination of the transmission path are the main points for COVID-19 surveillance (Brockwell, J. P., \& Davis, A. R.1996); However, because of the lack of visible clinical signs and inadequate knowledge of prevention that leads to COVID-19's rapid spread, most asymptomatic infections do not seek medical attention (Gao, W.J., Zheng, K., Ke, J., and Li, L.M., 2020). Hospitals should provide a robust preparedness plan for COVID patients to cope with the increase in healthcare demand. The balance of request (e.g., patients) and supply (e.g., resources) is an important principle that should be integrated with the preoperational plans(Ali, I., and Omar M L A., 2020). The allocation of resources within the minimum time is a vital part of any preparedness plant. Queuing theory provides full application in assessing the time spent by the user in the system and time elapsed in waiting to avail of the service.

The computer networks field has been found to exhibit self-similarity, and it plays a prominent role in the design of such networks (M. Gospodinov and E. Gospodinova,2005). Unlike the other models to represent network traffic, self-similarity-based models are best suited to represent real network properties such as burst network traffic, etc... To accommodate the history of contacts in the intelligent decision making of future routing protocols, 
we identify the opportunistic networking system to possess two high-level properties of predictability and connectedness, which determine the connectivity of the network. For the nodes participating in the opportunistic data exchanges to estimate their predictability and connectedness of contacts with their neighbors, they can utilize the self-similarity present in the network connectivity.

\subsection{Fundamentals of Self-similarity and Hurst Index Parameter:}

Day today's mathematics usually addresses that a self-similar thing is precisely or roughlysimilar to a part of itself (i.e., the entire has the same shape as one or more details). Numerous objects in the real globe, such as coastlines, are statistically self-similar: parts of them show the same statistical properties at multiple scales. Self-similarity is a typical characteristic of fractals. Also, Scale invariance is an exact form of self-similarity, where at any magnification, there is a smaller piece of the object that is similar to the whole. Take an example like, a side of the Koch snowflake is both symmetrical and scale-invariant; however, by not changing shape, it can be continually magnified 3x. Hence, non-trivial resemblance manifest in fractals is illustrated by their acceptable arrangement or detail on logically small scales. Because of counterexample, while any portion of a straight line may look like the whole, further detail is not revealed. Hence, the time just beginning phenomenon is said to show self-similarity if the numerical value of certainly visible quantity $f(x, t)$ calculated at diverse times, are different, but the dimensionless matching size at a known value of $x / t^{2}$ stay invariant. However, if the quantity $f(x, t)$ exhibits dynamic scaling, then it will happen. Basic information is just an additionto the idea of the resemblance of two triangles. Also, two triangles are alike if the numerical values of their sides are different. Hence the equivalent dimensionless quantities, such as their angles, agree.

\subsubsection{Mathematical Definition of self-similarity:}

Definition (1): A stochastic process $\{x(t), t \geq 0\}$ is known as self-similar if whichever

a> 0 , there subsists $b>0$ such that

$$
X(a t)=d\{b X(t)\}
$$

Let d denotes the parity of finite-dimensional distributions.

Definition (2): Arrival patterns are modelled as a point process. Assume that $X=\left\{X_{t} / \mathrm{t}=1,2 \ldots\right\}$ are the arrivals in the interval, segregate the time axis into disjoint intervals of unit length. Let $X$ be a second-order stationary process with autocorrelation function $\gamma(k) ; k \geq 0$ and variance $\sigma^{2}$ is given by

$$
\gamma(k)=\frac{\operatorname{Cov}\left(X_{t}, X_{t+k}\right)}{\operatorname{Var}\left(X_{t}\right)}
$$

The process ' $X$ ' is known to be precisely second-order self-similar with the Hurst index $H$ and variance $\sigma^{2}$ if

$$
\gamma(k)=\frac{\sigma^{2}}{2}\left[(k+1)^{2 H}-2 k^{2 H}+(k-1)^{2 H}\right], \forall \mathrm{k} \geq 1
$$

Definition (3): For each $m=1,2,3, \ldots$ assume a new time series as $X^{(m)}=\left\{X_{t}^{(m)} / \mathrm{t}=1,2, \ldots\right\}$

is determined averaging the unique time series process $X$ over non-overlapping

blocks of size $m$. i.e. $\quad X_{t}^{(m)}=\frac{1}{m} \sum_{i=1}^{m} \mathrm{x}_{(\mathrm{t}-1) \mathrm{m}+\mathrm{i}}, \mathrm{t}=1,2, \ldots \ldots$ 
For each $m$, this new series $X_{t}^{(m)}$, is also a second-order stationary process with ACF of $\gamma^{(m)}(k)$. Hurst index $\mathrm{H}$ and variance if the process ' $X$ ' is said to be asymptotically second-order self-similar with in terms of variance of the averaged process, we describe the similar method as

$$
\gamma^{(m)}(k)=\frac{\sigma^{2}}{2}\left[(k+1)^{2 H}-2 k^{2 H}+(K-1)^{2 H}\right], \forall k \geq 1
$$

Explaining and interpreting are very difficult for quantifying the self-similarity in mathematics, considering its complexity. But Hurst's (Hurst, 1951) constraint is enchanting because it addresses a lot of mathematics areas: autocorrelation, fractals, wavelets, etc. Hence Hurst catalog proposes a widespread calculation, whether a time series has LRD or not. This has been useful in the examination of system traffic study and modeling. But Hurst index $\mathrm{H}$ was employed to approximate the self-similarity. The Hurst exponent $\mathrm{H}$ was named later than the hydrologist, who depleted so many years to examine the difficulty of storage of the water concludes the altitude forms of the Nile River. Here, the range of Hurst exponent is $0.5 \leq H<1$. Also, quantifying exponent $H$ is a difficult task. Genuinely, many techniques for quantifying Hurst exponent show significantly diverse consequences\& other methods were rescaled accustomed range method and correlogram method. Despite very theoretical foundations, the practical application of these methods depends on the reviewed case's nature. The calculation is expensive in terms of computational power and time.

\subsubsection{Methods for computing Hurst Index:}

Hurst exponent $(\mathrm{H})$ is the classical parameter of measuring the intensity of self-similarity. The development of Hurst is traced before in 1951. The hydrologist H.E (Hurst H 2005 ) with his team investigates the optimum dam sizing of water storage and determines the Nile River's drought conditions. The Hurst parameter is used in the Financial market to make decisions about trading securities. It can also be applied in ecology to increase and decrease populations. The parameter has a range of $0.5<\mathrm{H}<1$ is a measure of self-similarity. There are several methods for Hurst index evaluation in a time-series (Roughness 2003). There are various methods for estimating Hurst exponent which provides the measurement of self-similar characteristic are as follows:

Method of Rescaled adjusted range statistics, (Hurst, 1978).

(ii) Variance time method, (Cox and smith,1953)

(iii) Periodogram method, (Daniell, 1948)

(iv) Correlogram method (Licklider, 1951

(v) Higuchi's method, (Higuchi, 1988)

\subsection{Description of $\mathrm{M} / \mathrm{M} / 1$ model:}

Waiting in line to get the appointment, check-up, and treatment is the typical scenario in India. The delay in getting treatment is the most crucial time for any patient. Nearly all of us waited for days or weeks before we had a medical appointment or scheduled treatment, and we hoped for something more when we arrived. In the hospital, patients waiting for beds are not uncommon in India, and there are frequent delays in surgery or medical tests. This delay has become more longer to the spreading of Corona disease and declared a pandemic by WHO. The basic principle of queue models gives a different type of resources to make sure that the patient procedure in a hospital or another health care facility is planned practically (Nosek Jr, R. A., and Wilson, J. P, 2001).Also, due to these models' orientation to maximize or reduce, is the time spent in queues waiting for treatment, thus escalating the efficiency of the whole care method. The problems of such queuing models are that: (1) these models are typically non-linear, which makes it challenging to create an empirical model for a wide variety of stochastic simulations of the patient arrival and treatment procedures, thus requiring simulation to validate and apply this model to actual patient therapy processes; (2) These models are based on a system stationary stage, i.e., on the stabilization of the design features over time. 


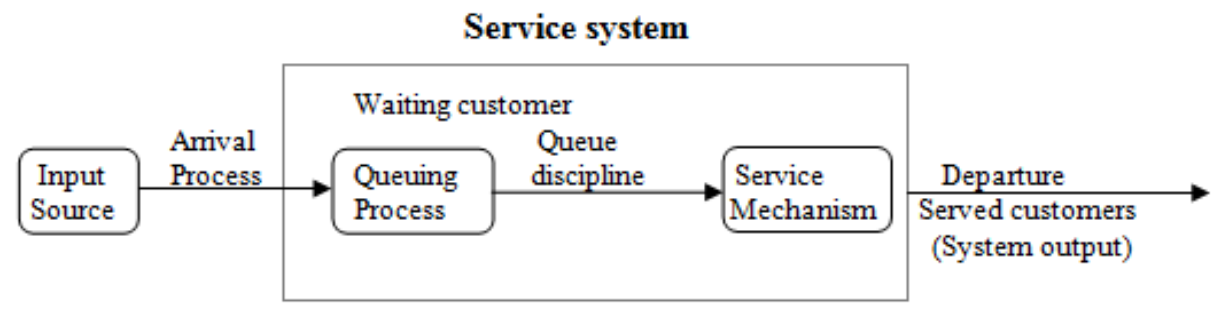

Fig-1: Service Model M/M/1

The structure of the queuing model is defined as

- $\quad$ input or arrival distribution,

- $\quad$ service distribution,

- $\quad$ service channels,

- maximum number of customers/objects in the system,

- $\quad$ population size or calling source,

- $\quad$ service discipline

- Input or arrival distribution: This corresponds to the pattern in which the patrons or things arrive at a service center. Arrivals are signified by the inter-arrival time, which means the time epoch among two consecutive appearances.

\section{Materials and methods}

In this situation, the Government of India introduced a rapid test to know the results of COVID-19 patients; under this our local area, government hospitals started rapid testing with help of Healthcare organizations where more COVID-19 expected people could test and get results within a day.

The concept of self-similarity was pioneered and is used to support modeling Geological and Hydrological problems. Self-similarity is a word where an entity's convinced property is kept concerning scale in time and space. If an entity is self-similar to its parts, a magnified bear a resemblance to the whole shape. (Meng, Q., Khoo, H.L.,2009) examined the patient's arrival to the health care center and observed that the patients waiting in queue to test COVID-19 and patients who tested confirmed results showed self-similarity characteristics.

These findings, from motivation, conclude that the arrival pattern of the testing center can be characterized as a self-similar process. Also, this study's very purpose is the quantifying Hurst exponent, addressing the intensity of self-similarity. The primary purpose of this research checked the behavior of real-life data pattern, is self-similar (Meng, Q., Khoo, H.L,2009), which is an addition to examine the presentation metrics, as mean waiting time and mean queue length against the traffic intensity. Hence research efforts, to arrive at a balance mean size of the queue and waiting time distribution for $\mathrm{M} / \mathrm{M} / 1$ Queuing systems. However, the queuing system structure is cleared as input or arrival distribution, service distribution, checking channels, the severe number of patrons in the scheme, people size or calling source, service obedience.

\subsection{Data of Covid-19 patients Collected from Primary Health Center}

Data is collected from the Health care center of Hospital for three months, July-2020 to September-2020, i.e., 92 days, Table-1.Using this data checking how the queuing theory is working with a single server, with patients' waiting time. We calculated patients waiting time, traffic intensity and tested that the self-similarity is existing to that data. And calculated Hurst parameter using different methods like rescaled adjusted range method and correlogram method. Collected data and sorted out as day wise as Table-2. 
Table-1: Data collected to test sample of COVID-19 from Healthcare center

\begin{tabular}{|c|c|c|c|c|c|c|c|c|c|c|c|c|c|c|c|c|c|c|c|c|c|c|}
\hline 4 & A & B & C & D & $E$ & $\mathrm{~F}$ & G & $\mathrm{H}$ & I & $\mathrm{J}$ & $\mathrm{K}$ & $\mathrm{L}$ & M & $\mathrm{N}$ & 0 & $\mathrm{P}$ & $Q$ & $\mathrm{R}$ & S & $\mathrm{T}$ & U & W \\
\hline 1 & $\begin{array}{l}\text { Laboratory } \\
\text { Name }\end{array}$ & Patient ID & \begin{tabular}{|l|} 
Patient \\
Name
\end{tabular} & Age & & $\begin{array}{l}\text { Contact } \\
\text { Number } \\
\text { Belongs } \\
\text { To }\end{array}$ & \begin{tabular}{|l} 
State \\
of \\
Reside \\
nce
\end{tabular} & $\begin{array}{l}\text { Distr } \\
\text { ict } \\
\text { of } \\
\text { Resi } \\
\text { den } \\
\text { ce }\end{array}$ & \begin{tabular}{|l} 
\\
Villa \\
ge \\
To \\
wn \\
\end{tabular} & $\begin{array}{l}\text { Was } \\
\text { the } \\
\text { patie } \\
\text { nt } \\
\text { quar } \\
\text { antin } \\
\text { ed }\end{array}$ & $\begin{array}{l}\text { Date of Sample } \\
\text { Collection }\end{array}$ & $\begin{array}{l}\text { Date of Sample } \\
\text { Received }\end{array}$ & $\begin{array}{l}\text { Sam } \\
\text { ple } \\
\text { Type }\end{array}$ & $\begin{array}{c}\text { Ho } \\
\text { spi } \\
\text { tali } \\
z e \\
d\end{array}$ & $\begin{array}{l}\text { Hospit } \\
\text { alizati } \\
\text { on } \\
\text { Date }\end{array}$ & $\begin{array}{l}\text { Sy } \\
\mathrm{mp} \\
\text { to } \\
\mathrm{ms} \\
\text { Sta } \\
\text { tus }\end{array}$ & $\begin{array}{l}\text { sympt } \\
\text { oms }\end{array}$ & $\begin{array}{l}\text { Testi } \\
\text { ng Kit } \\
\text { Used }\end{array}$ & $\begin{array}{l}\text { Repe } \\
\text { at } \\
\text { Sampl } \\
\text { e }\end{array}$ & $\begin{array}{l}\text { Date of Sample } \\
\text { Tested }\end{array}$ & $\begin{array}{c}\text { Final } \\
\text { Result } \\
\text { Sample }\end{array}$ & \\
\hline 2 & H B Halli Gh & $2.95 \mathrm{E}+12$ & MOHAl & 3 years & $M$ & relative & KARNA & $\mathrm{BAL}$ & $\mathrm{HBH}$ & No & $01-08-202010: 23$ & |01-08-2020 14:17| & Thro & No & N/A & Sy & fever & $\mathrm{Ag}-\mathrm{S}$ & No & $01-08-202014: 17$ & \multicolumn{2}{|c|}{ Antigen Negative } \\
\hline 3 & H B Halli Gh & $2.95 \mathrm{E}+12$ & HANUIS & 50 years & $M$ & patient & KARNA & BAL & $\mathrm{HBH}$ & No & 01-08-2020 10:30 & 01-08-2020 14:18 & Thro & No & N/A & Sy & fever & $\mathrm{Ag}-\mathrm{S}$ & No & 01-08-2020 14:18 & \multicolumn{2}{|c|}{ Antigen Positive } \\
\hline 4 & H B Halli Gh & $2.95 E+12$ & SARAS & 35 years & $F$ & relative & KARNA & BAL & $\mathrm{HBH}$ & No & 01-08-2020 10:33 & 01-08-2020 14:18 & Thro & No & N/A & Sy & fever & $\mathrm{Ag}-\mathrm{S}$ & No & 01-08-2020 14:18 & \multicolumn{2}{|c|}{ Antigen Negative } \\
\hline 5 & H B Halli Gh & $2.95 \mathrm{E}+12$ & GANES & 30 years & $M$ & patient & KARNA & $\mathrm{BAL}$ & $\mathrm{HBH}$ & No & $01-08-202010: 38$ & 01-08-2020 14:19 & Thro & No & N/A & Sy & fever & $\mathrm{Ag}-\mathrm{S}$ & No & $01-08-202014: 19$ & \multicolumn{2}{|c|}{ Antigen Negative } \\
\hline 6 & H B Halli Gh & $2.95 \mathrm{E}+12$ & BASAY & 41 years & $M$ & patient & KARNA & $\mathrm{BAL}$ & $\mathrm{HBH}$ & No & $01-08-202010: 42$ & 01-08-2020 14:20 & Thro & No & N/A & Sy & fever & $\mathrm{Ag}-\mathrm{S}$ & No & $01-08-202014: 20$ & \multicolumn{2}{|c|}{ Antigen Negative } \\
\hline 7 & H B Halli Gh & $2.95 E+12$ & HALAM & 35 years & $F$ & relative & KARNA & $\mathrm{BAL}$ & $\mathrm{HBH}$ & No & 01-08-2020 10:47 & 01-08-2020 14:21 & Thro & No & N/A & $\mathrm{As}$ & & $A g-S$ & No & 01-08-2020 14:21 & \multicolumn{2}{|c|}{ Antigen Negative } \\
\hline 8 & H B Halli Gh & $2.95 \mathrm{E}+12$ & JYOTH & 24 years & $\mathrm{F}$ & patient & KARNA & $\mathrm{BAL}$ & $\mathrm{HBH}$ & No & 01-08-2020 10:51 & 01-08-2020 14:22 & Thro & No & N/A & $\mathrm{As}$ & & $A g-S$ & No & 01-08-2020 14:22 & \multicolumn{2}{|c|}{ Antigen Negative } \\
\hline 9 & \begin{tabular}{l|l}
$\ldots$ &
\end{tabular} & \begin{tabular}{|l|l}
$\ldots$ & \\
\end{tabular} & $\ldots$ & $\ldots$ & & $\ldots$ & $\ldots$ & \begin{tabular}{|l|}
$\ldots$ \\
\end{tabular} & .... & $\ldots$ & \begin{tabular}{|l|}
$\ldots$ \\
\end{tabular} & \begin{tabular}{|l|}
$\ldots$ \\
\end{tabular} & \begin{tabular}{l|l}
$\ldots$. &.
\end{tabular} & $\ldots . .$. & $\ldots$ & \begin{tabular}{l|l}
$\ldots$. &
\end{tabular} & $\ldots$ & $\ldots$ & $\ldots$ & $\ldots$ & $\ldots$ & \\
\hline 10 & $\ldots$ & $\ldots$ & $\ldots$ & $\ldots$ & & $\ldots$ & $\ldots$ & $\ldots$ & $\ldots$ & $\ldots$ & $\ldots$ & $\ldots$ & $\ldots$ & \begin{tabular}{l|l}
$\ldots$. & \\
\end{tabular} & $\ldots$ & \begin{tabular}{l|l}
$\ldots .$. &. \\
\end{tabular} & $\ldots$ & $\ldots$ & $\ldots$ & $\ldots$ & $\ldots$ & \\
\hline
\end{tabular}

Table-2: Day-wise sample Arrivals of COVID-19 positive cases to analyze Self Similarity and Hurst Parameter

\begin{tabular}{|c|c|c|c|c|c|c|c|c|}
\hline S.No & Date & Hours & Time & $\begin{array}{c}\text { No.of } \\
\text { patients } \\
\text { sample } \\
\text { collected }\end{array}$ & $\begin{array}{c}\text { Sample } \\
\text { type } \\
\text { Throat } \\
\text { swab }\end{array}$ & $\begin{array}{l}\text { Sample } \\
\text { type } \\
\text { Nasoph } \\
\text { aryngea } \\
\text { 1 swab }\end{array}$ & $\begin{array}{l}\text { confirmed } \\
\text { cases }\end{array}$ & $\begin{array}{c}\text { Negative } \\
\text { Cases }\end{array}$ \\
\hline 1 & 01-07-2020 & 3Hours & 10AM -2PM & 30 & 30 & 0 & 2 & 28 \\
\hline 2 & 02-07-2020 & 3 Hours & 11PM-4PM & 21 & 21 & 0 & 3 & 19 \\
\hline 3 & 03-07-2020 & 4 Hours & 10PM-2PM & 42 & 42 & 0 & 2 & 40 \\
\hline 4 & 04-07-2020 & 1 Hour & 2Pm-3PM & 16 & 16 & 0 & 0 & 16 \\
\hline 5 & $05-07-2020$ & 2 Hours & 1 PM-3 PM & 28 & 28 & 0 & 1 & 27 \\
\hline 6 & 06-07-2020 & 2 Hours & 10 PM-12 PM & 15 & 15 & 0 & 0 & 15 \\
\hline 7 & 07-07-2020 & 3Hours & 11 PM-3 PM & 34 & 34 & 0 & 2 & 32 \\
\hline 8 & 08-07-2020 & 2 Hours & 10AM-12PM & 20 & 20 & 0 & 1 & 19 \\
\hline 9 & 09-07-2020 & 2 Hours & 10AM-12PM & 15 & 15 & 0 & 1 & 14 \\
\hline 10 & $10-07-2020$ & 2 Hours & 10AM -12PM & 17 & 17 & 0 & 1 & 16 \\
\hline 11 & $11-07-2020$ & 3 Hours & 1PM-3PM & 31 & 31 & 0 & 2 & 29 \\
\hline 12 & $12-07-2020$ & 1 Hour & 1PM-2PM & 14 & 14 & 0 & 0 & 14 \\
\hline 13 & $13-07-2020$ & 1 Hour & 2PM-3PM & 26 & 26 & 0 & 2 & 24 \\
\hline 14 & 14-07-2020 & 1 Hour & 1PM-2PM & 18 & 18 & 0 & 1 & 17 \\
\hline 15 & $15-07-2020$ & 2 Hours & 2PM-4PM & 22 & 22 & 0 & 1 & 21 \\
\hline 16 & $16-07-2020$ & 3 Hours & 12PM-4PM & 35 & 35 & 0 & 3 & 32 \\
\hline 17 & $17-07-2020$ & 3Hours & 10AM-3PM & 25 & 25 & 0 & 2 & 23 \\
\hline 18 & $18-07-2020$ & 3 Hours & 1PM-4PM & 36 & 26 & 10 & 6 & 30 \\
\hline 19 & 19-07-2020 & 2Hours & 2AM-4PM & 18 & 16 & 2 & 1 & 17 \\
\hline 20 & 20-07-2020 & 4 Hours & 10AM-4PM & 60 & 60 & 0 & 0 & 60 \\
\hline$\ldots$. & $\ldots$ & $\ldots$ & $\ldots$ & $\ldots$. & $\ldots$. & $\ldots$. & $\ldots$. & $\ldots$. \\
\hline$\ldots$. & $\ldots$ & $\ldots$. & $\ldots$. & $\ldots$. & $\ldots$. & $\ldots$. & $\ldots$. & $\ldots$. \\
\hline$\ldots$ & $\ldots$ & $\ldots$ & $\ldots$ & $\ldots$. & $\ldots$. & $\ldots$. & $\ldots$ & $\ldots$ \\
\hline$\ldots$ & $\ldots$ & $\ldots$ & $\ldots$ & $\ldots$. & $\ldots$ & $\ldots$ & $\ldots$ & $\ldots$ \\
\hline 92 & $30-09-2020$ & 3Hours & 10AM-1PM & 41 & 0 & 41 & 7 & 34 \\
\hline
\end{tabular}


Table-3: Hurst index measure for patients' arrivals by R/S Method

\begin{tabular}{|c|c|c|c|c|c|c|c|}
\hline $\begin{array}{c}\text { Day of } \\
\text { Test }\end{array}$ & $\begin{array}{c}\text { Day wise } \\
\text { No.of } \\
\text { Arrivals }\end{array}$ & Log (Time) & $\log (\mathrm{R} / \mathrm{S})$ & $\begin{array}{l}\text { Day } \\
\text { of } \\
\text { Test }\end{array}$ & $\begin{array}{c}\text { Day wise } \\
\text { No.of } \\
\text { Arrivals }\end{array}$ & Log(Time) & $\log (\mathrm{R} / \mathrm{S})$ \\
\hline 1 & 30 & 0.47712125 & 0.13114085 & 46 & 13 & 1.68124124 & 1.08550072 \\
\hline 2 & 21 & 0.60205999 & 0.22135452 & 47 & 15 & 1.69019608 & 1.09173373 \\
\hline 3 & 42 & 0.69897 & 0.28367455 & 48 & 41 & 1.69897 & 1.09875498 \\
\hline 4 & 16 & 0.77815125 & 0.3326208 & 49 & 31 & 1.70757018 & 1.10602652 \\
\hline 5 & 28 & 0.84509804 & 0.38773778 & 50 & 27 & 1.71600334 & 1.11261314 \\
\hline 6 & 15 & 0.90308999 & 0.43256918 & 51 & 40 & 1.72427587 & 1.11989167 \\
\hline 7 & 34 & 0.95424251 & 0.47365787 & 52 & 31 & 1.73239376 & 1.12704759 \\
\hline 8 & 20 & 1 & 0.50948845 & 53 & 15 & 1.74036269 & 1.133535 \\
\hline 9 & 15 & 1.04139269 & 0.54303708 & 54 & 12 & 1.74818803 & 1.14017333 \\
\hline 10 & 17 & 1.07918125 & 0.57415166 & 55 & 37 & 1.75587486 & 1.147807 \\
\hline 11 & 31 & 1.11394335 & 0.6005414 & 56 & 38 & 1.76342799 & 1.15529214 \\
\hline 12 & 14 & 1.14612804 & 0.62610735 & 57 & 40 & 1.77085201 & 1.16324136 \\
\hline 13 & 26 & 1.17609126 & 0.64925406 & 58 & 38 & 1.77815125 & 1.17133642 \\
\hline 14 & 18 & 1.20411998 & 0.67007913 & 5960 & 45 & 1.78532984 & 1.18010859 \\
\hline 15 & 22 & 1.23044892 & 0.68912623 & 61 & 40 & 1.79239169 & 1.18800983 \\
\hline 16 & 35 & 1.25527251 & 0.70694939 & 62 & 26 & 1.79934055 & 1.19598055 \\
\hline 17 & 25 & 1.2787536 & 0.72234925 & 63 & 59 & 1.80617997 & 1.20430306 \\
\hline 18 & 36 & 1.30103 & 0.73863366 & 64 & 52 & 1.81291336 & 1.21208432 \\
\hline 19 & 18 & 1.32221929 & 0.7534145 & 65 & 46 & 1.81954394 & 1.21918183 \\
\hline 20 & 60 & 1.34242268 & 0.7708074 & 67 & 55 & 1.8260748 & 1.22773749 \\
\hline 21 & 11 & 1.36172784 & 0.78797258 & 68 & 54 & 1.83250891 & 1.23664502 \\
\hline 22 & 11 & 1.38021124 & 0.806093 & 69 & 42 & 1.83884909 & 1.24572148 \\
\hline 23 & 6 & 1.39794001 & 0.82415429 & 70 & 21 & 1.84509804 & 1.25523595 \\
\hline 24 & 8 & 1.41497335 & 0.8423439 & 71 & 75 & 1.85125835 & 1.26452441 \\
\hline 25 & 13 & 1.43136376 & 0.86016132 & 72 & 80 & 1.8573325 & 1.27316119 \\
\hline 26 & 54 & 1.44715803 & 0.87790441 & 73 & 54 & 1.86332286 & 1.2804125 \\
\hline 27 & 29 & 1.462398 & 0.89481683 & 74 & 78 & 1.86923172 & 1.28831851 \\
\hline 28 & 96 & 1.47712125 & 0.91118905 & 75 & 75 & 1.87506126 & 1.29568404 \\
\hline 29 & 32 & 1.49136169 & 0.92613078 & 76 & 47 & 1.88081359 & 1.30314977 \\
\hline 30 & 44 & 1.50514998 & 0.94056538 & 77 & 15 & 1.88649073 & 1.31077449 \\
\hline 31 & 39 & 1.51851394 & 0.95355241 & 78 & 54 & 1.8920946 & 1.31843252 \\
\hline 32 & 36 & 1.53147892 & 0.96649657 & 79 & 75 & 1.89762709 & 1.32515071 \\
\hline 33 & 18 & 1.54406804 & 0.97778441 & 80 & 65 & 1.90308999 & 1.33183375 \\
\hline 34 & 54 & 1.5563025 & 0.98921775 & 81 & 21 & 1.90848502 & 1.33853622 \\
\hline 35 & 27 & 1.56820172 & 0.99937899 & 82 & 89 & 1.91381385 & 1.34453728 \\
\hline 36 & 35 & 1.5797836 & 1.00902942 & 83 & 69 & 1.91907809 & 1.35043427 \\
\hline 37 & 30 & 1.59106461 & 1.01861399 & 84 & 43 & 1.92427929 & 1.35532636 \\
\hline 38 & 33 & 1.60205999 & 1.02726765 & 85 & 82 & 1.92941893 & 1.35883631 \\
\hline
\end{tabular}




\begin{tabular}{|l|l|l|l|l|l|l|l|}
39 & 19 & 1.61278386 & 1.03554449 & 86 & 75 & 1.93449845 & 1.36162661 \\
\hline 40 & 12 & 1.62324929 & 1.04256949 & 87 & 52 & 1.93951925 & 1.36325591 \\
\hline 41 & 32 & 1.63346846 & 1.05048767 & 88 & 113 & 1.94448267 & 1.37353417 \\
\hline 42 & 14 & 1.64345268 & 1.0578121 & 89 & 270 & 1.94939001 & 1.38305951 \\
\hline 43 & 27 & 1.65321251 & 1.06491877 & 90 & 149 & 1.95424251 & 1.39181331 \\
\hline 44 & 36 & 1.66275783 & 1.07168145 & 91 & 93 & 1.95904139 & 1.39983598 \\
\hline 45 & 28 & 1.67209786 & 1.07942745 & 92 & 104 & 1.96378783 & 1.40334725 \\
\hline
\end{tabular}

Table-4Hurst parameter for No.of patient Arrivals per day Using Correlogram Method

\begin{tabular}{|c|c|c|c|c|c|c|c|}
\hline & \multirow{2}{*}{ Lag } & \multirow{2}{*}{$\begin{array}{c}\text { Autocor } \\
\text { relation }\end{array}$} & Std. Error & \multicolumn{2}{|c|}{ Box-Ljung Statistic } & \multirow{2}{*}{ Log SNO } & \multirow{2}{*}{ Log Sample ACF } \\
\cline { 4 - 6 } & .575 & .103 & 31.437 & 1 & .000 & 0 & -0.240219397 \\
2 & .407 & .102 & 47.364 & 2 & .000 & 0.30103 & -0.390263454 \\
3 & .453 & .101 & 67.304 & 3 & .000 & 0.477121 & -0.343896443 \\
4 & .473 & .101 & 89.305 & 4 & .000 & 0.60206 & -0.32500331 \\
5 & .188 & .100 & 92.815 & 5 & .000 & 0.69897 & -0.726046949 \\
6 & .218 & .100 & 97.607 & 6 & .000 & 0.778151 & -0.660951346 \\
7 & .278 & .099 & 105.481 & 7 & .000 & 0.845098 & -0.555642978 \\
8 & .196 & .099 & 109.447 & 8 & .000 & 0.90309 & -0.707144128 \\
9 & .132 & .098 & 111.257 & 9 & .000 & 0.954243 & -0.880036932 \\
10 & .222 & .097 & 116.473 & 10 & .000 & 1 & -0.652875715 \\
11 & .182 & .097 & 120.017 & 11 & .000 & 1.041393 & -0.739487192 \\
12 & .035 & .096 & 120.147 & 12 & .000 & 1.079181 & -1.459146496 \\
13 & .115 & .096 & 121.595 & 13 & .000 & 1.113943 & -0.93938446 \\
14 & .199 & .095 & 126.003 & 14 & .000 & 1.146128 & -0.700251622 \\
15 & .170 & .094 & 129.235 & 15 & .000 & 1.176091 & -0.770499055 \\
16 & .088 & .094 & 130.122 & 16 & .000 & 1.20412 & -1.054003291 \\
\hline
\end{tabular}

Table-5: Average Queue Length for various Hurst parameters

\begin{tabular}{|c|c|c|c|c|c|c|}
\hline $\begin{array}{c}\text { Day } \\
\text { of } \\
\text { Test }\end{array}$ & $\begin{array}{c}\text { Patients } \\
\text { Arrival } \\
\lambda\end{array}$ & $\begin{array}{c}\text { Patients } \\
\text { testing } \\
\text { (Service) } \mu\end{array}$ & $\begin{array}{c}\text { Traffic } \\
\text { Intensity } \\
\mathrm{P}=\lambda / \mu\end{array}$ & $\begin{array}{c}\text { Queue } \\
\text { Length }\end{array}$ & $\begin{array}{c}\mathrm{H}=0.88 \\
\rho^{0.5 /(1-\mathrm{H})}\end{array}$ & $\begin{array}{c}\mathrm{H}=0.82 \\
\rho^{0.5 /(1-\mathrm{H})}\end{array}$ \\
\hline 1 & 30 & 45 & 0.6667 & 1.3333 & 26.178 & 15.516 \\
\hline 2 & 21 & 38 & 0.5526 & 0.6827 & 21.694 & 12.945 \\
\hline 3 & 42 & 78 & 0.5385 & 0.6282 & 21.316 & 12.723 \\
\hline 4 & 16 & 19 & 0.8421 & 4.4912 & 46.291 & 26.565 \\
\hline 5 & 28 & 39 & 0.7179 & 1.8275 & 29.489 & 17.376 \\
\hline 6 & 15 & 30 & 0.5000 & 0.5000 & 20.433 & 12.201 \\
\hline 7 & 34 & 45 & 0.7556 & 2.3354 & 32.811 & 19.222 \\
\hline 8 & 20 & 23 & 0.8696 & 5.7971 & 54.123 & 30.750 \\
\hline 9 & 14 & 17 & 0.8235 & 3.8431 & 42.323 & 24.424 \\
\hline 10 & 17 & 19 & 0.8947 & 7.6053 & 64.686 & 36.325 \\
\hline
\end{tabular}




\begin{tabular}{|c|c|c|c|c|c|c|}
\hline 11 & 31 & 80 & 0.3875 & 0.2452 & 18.832 & 11.208 \\
\hline 12 & 14 & 22 & 0.6364 & 1.1136 & 24.679 & 14.665 \\
\hline 13 & 26 & 43 & 0.6047 & 0.9248 & 23.376 & 13.919 \\
\hline 14 & 18 & 23 & 0.7826 & 2.8174 & 35.902 & 20.925 \\
\hline 15 & 22 & 25 & 0.8800 & 6.4533 & 57.990 & 32.800 \\
\hline 16 & 35 & 77 & 0.4545 & 0.3788 & 19.625 & 11.713 \\
\hline 17 & 25 & 40 & 0.6250 & 1.0417 & 24.184 & 14.382 \\
\hline 18 & 36 & 47 & 0.7660 & 2.5068 & 33.916 & 19.833 \\
\hline 19 & 18 & 21 & 0.8571 & 5.1429 & 50.224 & 28.673 \\
\hline 20 & 60 & 68 & 0.8824 & 6.6176 & 58.952 & 33.308 \\
\hline 21 & 52 & 64 & 0.8125 & 3.5208 & 40.326 & 23.340 \\
\hline 22 & 12 & 16 & 0.7500 & 2.2500 & 32.257 & 18.916 \\
\hline 23 & 6 & 7 & 0.8571 & 5.1429 & 50.224 & 28.673 \\
\hline 24 & 8 & 11 & 0.7273 & 1.9394 & 30.227 & 17.788 \\
\hline 25 & 13 & 17 & 0.7647 & 2.4853 & 33.778 & 19.757 \\
\hline 26 & 54 & 73 & 0.7397 & 2.1024 & 31.296 & 18.383 \\
\hline 27 & 29 & 44 & 0.6591 & 1.2742 & 25.777 & 15.289 \\
\hline 28 & 96 & 112 & 0.8571 & 5.1429 & 50.224 & 28.673 \\
\hline 29 & 32 & 44 & 0.7273 & 1.9394 & 30.227 & 17.788 \\
\hline 30 & 44 & 52 & 0.8462 & 4.6538 & 47.277 & 27.095 \\
\hline 31 & 39 & 47 & 0.8298 & 4.0452 & 43.566 & 25.097 \\
\hline 32 & 36 & 46 & 0.7826 & 2.8174 & 35.902 & 20.925 \\
\hline 33 & 18 & 33 & 0.5455 & 0.6545 & 21.499 & 12.831 \\
\hline 34 & 54 & 110 & 0.4909 & 0.4734 & 20.252 & 12.093 \\
\hline 35 & 27 & 33 & 0.8182 & 3.6818 & 41.325 & 23.883 \\
\hline 36 & 35 & 44 & 0.7955 & 3.0934 & 37.649 & 21.882 \\
\hline 37 & 30 & 43 & 0.6977 & 1.6100 & 28.042 & 16.566 \\
\hline 38 & 33 & 82 & 0.4024 & 0.2710 & 18.970 & 11.300 \\
\hline 39 & 19 & 22 & 0.8636 & 5.4697 & 52.177 & 29.715 \\
\hline 40 & 12 & 20 & 0.6000 & 0.9000 & 23.204 & 13.820 \\
\hline 41 & 32 & 48 & 0.6667 & 1.3333 & 26.178 & 15.516 \\
\hline 42 & 18 & 26 & 0.6923 & 1.5577 & 27.692 & 16.370 \\
\hline 43 & 27 & 50 & 0.5400 & 0.6339 & 21.355 & 12.747 \\
\hline 44 & 36 & 58 & 0.6207 & 1.0157 & 24.004 & 14.280 \\
\hline 45 & 28 & 58 & 0.4828 & 0.4506 & 20.099 & 12.001 \\
\hline 46 & 13 & 30 & 0.4333 & 0.3314 & 19.326 & 11.527 \\
\hline
\end{tabular}




\begin{tabular}{|c|c|c|c|c|c|c|}
\hline 47 & 15 & 23 & 0.6522 & 1.2228 & 25.426 & 15.090 \\
\hline 48 & 41 & 44 & 0.9318 & 12.7348 & 93.431 & 51.184 \\
\hline 49 & 31 & 68 & 0.4559 & 0.3820 & 19.646 & 11.725 \\
\hline 50 & 27 & 49 & 0.5510 & 0.6763 & 21.649 & 12.919 \\
\hline 51 & 40 & 81 & 0.4938 & 0.4818 & 20.309 & 12.127 \\
\hline 52 & 31 & 37 & 0.8378 & 4.3288 & 45.302 & 26.033 \\
\hline 53 & 15 & 16 & 0.9375 & 14.0625 & 100.653 & 54.860 \\
\hline 54 & 12 & 13 & 0.9231 & 11.0769 & 84.300 & 46.506 \\
\hline 55 & 37 & 47 & 0.7872 & 2.9128 & 36.507 & 21.257 \\
\hline 56 & 38 & 43 & 0.8837 & 6.7163 & 59.528 & 33.612 \\
\hline 57 & 40 & 58 & 0.6897 & 1.5326 & 27.523 & 16.275 \\
\hline 58 & 38 & 53 & 0.7170 & 1.8164 & 29.415 & 17.335 \\
\hline 59 & 45 & 76 & 0.5921 & 0.8595 & 22.923 & 13.658 \\
\hline 60 & 40 & 52 & 0.7692 & 2.5641 & 34.284 & 20.036 \\
\hline 61 & 26 & 30 & 0.8667 & 5.6333 & 53.151 & 30.234 \\
\hline 62 & 59 & 78 & 0.7564 & 2.3489 & 32.898 & 19.270 \\
\hline 63 & 52 & 128 & 0.4063 & 0.2780 & 19.009 & 11.325 \\
\hline 64 & 46 & 49 & 0.9388 & 14.3946 & 102.448 & 55.770 \\
\hline 65 & 55 & 98 & 0.5612 & 0.7178 & 21.939 & 13.087 \\
\hline 66 & 54 & 65 & 0.8308 & 4.0783 & 43.770 & 25.206 \\
\hline 67 & 42 & 51 & 0.8235 & 3.8431 & 42.323 & 24.424 \\
\hline 68 & 21 & 22 & 0.9545 & 20.0455 & 132.395 & 70.810 \\
\hline 69 & 75 & 85 & 0.8824 & 6.6176 & 58.952 & 33.308 \\
\hline 70 & 80 & 125 & 0.6400 & 1.1378 & 24.844 & 14.759 \\
\hline 71 & 54 & 58 & 0.9310 & 12.5690 & 92.523 & 50.720 \\
\hline 72 & 78 & 83 & 0.9398 & 14.6602 & 103.881 & 56.497 \\
\hline 73 & 75 & 155 & 0.4839 & 0.4536 & 20.119 & 12.013 \\
\hline 74 & 47 & 51 & 0.9216 & 10.8284 & 82.920 & 45.795 \\
\hline 75 & 15 & 31 & 0.4839 & 0.4536 & 20.119 & 12.013 \\
\hline 76 & 54 & 65 & 0.8308 & 4.0783 & 43.770 & 25.206 \\
\hline 77 & 75 & 115 & 0.6522 & 1.2228 & 25.426 & 15.090 \\
\hline 78 & 65 & 110 & 0.5909 & 0.8535 & 22.882 & 13.634 \\
\hline 79 & 21 & 27 & 0.7778 & 2.7222 & 35.296 & 20.592 \\
\hline 80 & 89 & 97 & 0.9175 & 10.2075 & 79.456 & 44.009 \\
\hline 81 & 69 & 99 & 0.6970 & 1.6030 & 27.995 & 16.540 \\
\hline
\end{tabular}




\begin{tabular}{|l|c|c|c|c|c|c|}
82 & 43 & 60 & 0.7167 & 1.8127 & 29.391 & 17.322 \\
\hline 83 & 82 & 154 & 0.5325 & 0.6064 & 21.165 & 12.635 \\
\hline 84 & 75 & 86 & 0.8721 & 5.9461 & 55.004 & 31.219 \\
\hline 85 & 52 & 61 & 0.8525 & 4.9253 & 48.916 & 27.974 \\
\hline 86 & 113 & 150 & 0.7533 & 2.3007 & 32.586 & 19.098 \\
\hline 87 & 270 & 300 & 0.9000 & 8.1000 & 67.529 & 37.813 \\
\hline 88 & 149 & 160 & 0.9313 & 12.6142 & 92.771 & 50.847 \\
\hline 89 & 93 & 165 & 0.5636 & 0.7280 & 22.009 & 13.129 \\
\hline 90 & 104 & 135 & 0.7704 & 2.5845 & 34.415 & 20.108 \\
\hline 91 & 159 & 170 & 0.9353 & 13.5193 & 97.707 & 53.363 \\
\hline 92 & 41 & 43 & 0.9535 & 19.5465 & 129.791 & 69.513 \\
\hline
\end{tabular}

\subsection{Performance Measures for the Queue model M/M/1}

Here we analyze the model with an exponential distribution of inter-arrival times with a mean $1 / \omega$ and an exponential distribution of service times with mean $1 / v$ of a single server. The exponential distribution permits a straightforward definition position of the system with any time t. The service discipline of First Come First Served (FCFS) is accepted.

\section{Potential utilization of service facility $\rho$}

The potential utilization of service facilities is obtained by dividing the average arrival rate $\omega$ (in time) by the average service rate $v$.

$$
\text { i.e. } \rho=\frac{\omega}{v}
$$

Whenever the value of $\omega$ is more significant, the arrival of patients will increase, and therefore the system will work harder, and queue length will be longer. Subsequently, whenever the value of $\omega$ is lesser, the queue will be shorter, than the use of the system will be low. If a patient's arrival rate within the system is more than the service rate, i.e., $\omega>v$ then $\rho>1$, which suggests the system capability lesser, than the incoming patients, Hence the queue length is increased. About queueing system, the average arrival rate is lesser than the average service rate,

$$
\text { i.e., } \omega<v \text {. }
$$

The average number of patients either waiting in a queue or service:

The mean number of patients either waiting in a queue or service

$p_{j}=(p)(q)^{j}$, where $j=0,1,2, \ldots$.

Since the patients in the system follows the geometric distribution assume $j=0$, and

$p_{j}=(p)(q)^{j}$ where $p+q=1$

\section{Mean Number of Patients waiting in the queue or in service:}

The mean number of patients within a system is equal to the mean number of patients within the queue or service. As it can be defined as

$$
N=1 /(1-q)
$$




\section{Mean Waiting Time in Queue (Wq):}

The mean waiting time in the queue (before services are provided)is equal to the meantime in which a patient waits within the queue for getting service. Hence formula is

$$
W q=L q / \omega
$$

\section{Mean Time Spent in the System $\left(\mathbf{W}_{\mathrm{s}}\right)$ :}

The mean time spent in the system (on queue and getting service) is the same as the total time spent by a patient in a system includes the service time and waiting time. Hence formula is

$$
W_{s}=W q+1 / v
$$

\subsubsection{Rescaled Adjusted Range Statistics Method}

Self-similarity statistically signifies the statistical properties and is designed for the whole data sets. These are identical to each data set's sub-sections, which are relevant to measuring the Hurst parameter, where the Rescaled range statistics are calculated (Gospodinov, M., E. Gospodinova,2005) over divisions of various sizes. Fig-2 describes that the Rescaled range is computed for the whole data set $\left(R S a v e_{0}=R S_{0}\right)$. After that, it is calculated for bisects of two data sets, ensuing $R S_{0}$ and $R S_{1}$. Procedure persists by separating each one of preceding sections in half and manipulative rescaled range of each of the new and averaging each section's Rescaled range values. The subsection ends as the unit acquires very small (as a minimum of eight data points).

The Hurst index $\mathrm{H}$ is approximated by measuring the mean of rescaled range over several sections of data. Designed of available observations as a set, $X_{1}, X_{2, . .}, X_{n}$ by the sample mean $\mu=E\left[X_{i}\right]$ be described a series of regulated partial sums:

$$
W_{j}=\left(X_{1}+X_{2}+:: .+X_{j}\right)-j X(n), j=1,2,3 \ldots \ldots n
$$

Where $\bar{X}$ is the average of the first n number of observations.

$R(n)$ is the range described as

$R(n)=\max \left(0, W_{1}, W_{2, \ldots}, W_{n}\right)-\min \left(0, W_{1}, W_{2, \ldots}, W_{n}\right)$

$S(n)$ is the standard deviation of the observations $X_{1}, X_{2}, \ldots, X_{n}$ is defined as

$\mathrm{S}(\mathrm{n})=\sqrt{E\left(X_{i}-\mu\right)^{2}}$

The Hurst index be accessible through a rescaled adjusted range

$\mathrm{R} / \mathrm{S}$ statistics $=\mathrm{R}(\mathrm{n}) / \mathrm{S}(\mathrm{n})$

The predictable value of $R(n)=S(n)$ asymptotically gratify the power-law relation

$\mathrm{E}\left[\frac{R(n)}{S(n)}\right] \rightarrow c n^{H}$, as $\mathrm{n} \rightarrow \infty$,

Where a finite constant $c>0$ and $H>0.5$ is Hurst index.

Through a power function with an index of 0.5 , the predictable value will be described for the short range-model. $\mathrm{E}\left[\frac{R(n)}{S(n)}\right] \rightarrow d n^{0.5}$, as $\mathrm{n} \rightarrow \infty$,

\begin{tabular}{|c|c|c|c|c|c|c|c|c|c|}
\hline \multirow{3}{*}{$4 \overline{4786}$} & \multicolumn{8}{|c|}{$\mathrm{RS}_{0}$} & \multirow{2}{*}{$\begin{array}{l}\text { RSare }_{0} \\
\text { RSare }_{1}\end{array}$} \\
\hline & \multicolumn{4}{|c|}{$\mathrm{RS}_{0}$} & \multicolumn{4}{|c|}{$\mathrm{RS}_{1}$} & \\
\hline & \multicolumn{2}{|c|}{$\mathrm{RS}_{0}$} & \multicolumn{2}{|c|}{$\mathrm{RS}_{1}$} & \multicolumn{2}{|c|}{$\mathrm{RS}_{2}$} & \multicolumn{2}{|c|}{$\mathrm{RS}_{3}$} & RSare $_{2}$ \\
\hline & $\mathrm{RS}_{0}$ & RS1 & RS2 & RS3 & $\mathrm{RS}_{4}$ & $\mathrm{RS}_{5}$ & $\mathrm{RS}_{6}$ & $\mathbf{R S}_{7}$ & RSare $_{3}$ \\
\hline & \begin{tabular}{|l|l|}
$\mathrm{RS}_{0}$ & $\mathrm{RS}_{1}$
\end{tabular} & \begin{tabular}{l|l}
$\mathrm{RS}_{2}$ & $\mathrm{RS}$
\end{tabular} & \begin{tabular}{|l|l|}
$\mathrm{RS}_{4}$ & $\mathrm{RS}_{5}$
\end{tabular} & \begin{tabular}{|l|l|}
$\mathrm{RS}_{6}$ & $\mathrm{RS}_{7}$
\end{tabular} & \begin{tabular}{|l|l}
$\mathrm{RS}_{8}$ & $\mathrm{RS}$
\end{tabular} & \begin{tabular}{|l|l|}
$\mathrm{RS}_{10}$ & $\mathrm{RS}$ \\
\end{tabular} & $\mathrm{RS}_{12} \mid \mathrm{RS}$ & \begin{tabular}{l|l|l}
$\mathrm{RS}_{14}$ & $\mathrm{RS}$
\end{tabular} & $=\mathrm{RSare}_{4}$ \\
\hline
\end{tabular}

Where $d$ is the finite constant. The variation among Equation -15 and Equation-17 is called the Hurst effect. 


\section{Fig-2 R/S method}

\subsubsection{Correlogram Method}

The plot of Autocorrelation Function (ACF) in time series analysis is acknowledged like correlogram, where the approximated correlation can be specified in terms of ACF of $\gamma(k)$ as(Brockwell, J. P., \& Davis, A. R.1996)

$$
\rho(k)=\frac{\gamma(k)}{\gamma(0)}
$$

It is examined as slow rot of correlation, and proportional to $k^{2 H-2}$ for $0.5<H<1$ specify Long-memory process. As a result of the sample, ACF is supposed to show this property. An enhanced plot for performing LRD is the plot for Auto Correlation Function within the logarithmic scale. For long memory processes, if an asymptotic decay of correlation is hyperbolic, subsequently the points in the plot supposed to roughly scattered in the order of a line through a -ve slope of $2 \mathrm{H}-2$, the points must be inclined to diverse of minus infinity on an exponential tempo for short memory process. If the series has LRD, then the log-log correlogram is very helpful. Since a preface heuristic approach of data, it is a realistic one. Some difficulty about sample correlation exists, which is not as much recognized can be establishing (Beran, J., Taqqu, M.S. and Willinger, W, 1995). Although it is neither extensively used nor the striking process of assessment, the self-similarity measuring index $\mathrm{H}$ can be approximated with this method. Obtaining the form of an equation is

$$
\rho(k)=\hat{H}(2 \hat{H}-1) k^{2 \hat{H}-2}
$$

In this segment, we describe some numerical results of mean queue length $(\bar{L})$ against traffic intensity. For that, we use the formula (Gunther 2000) given under

$$
\bar{L}=\frac{\rho^{0.5 /(1-H)}}{(1-\rho)^{H /(1-H)}} .
$$

\section{Numerical results and discussion:}

Using Table-2 day-wise patients' arrival data of COVID-19 we analysed that the arrival pattern is having selfsimilarity expressed in Fig-3.And Using Table-2 day-wise COVID-19 cases confirmed patients have selfsimilarity, which is described in Fig-4.

Hurst index parameter Calculated for patients arrivals by R/S method we get 0.8824 by (PushpalathaSarla, Mallikarjuna Reddy, 2020) $\rho$ is the traffic intensity, Results are demonstrated in Table-3 and it's liner trend in Fig -5. By applying the Correlogram Method, we get 0.8195 expressed in Table-4.Its graphical representation trend figure is depicted in Fig-6. 


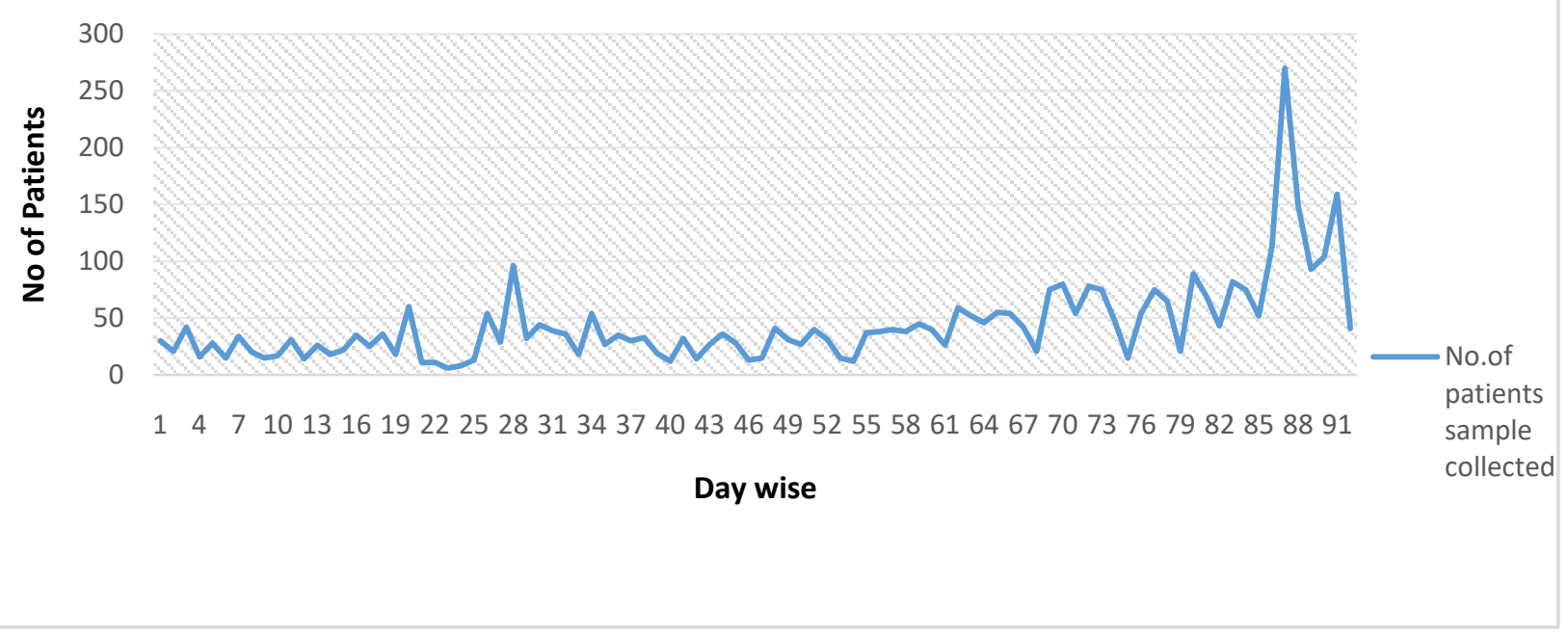

Fig-3: Self-similar Nature of Number of Patients arrival

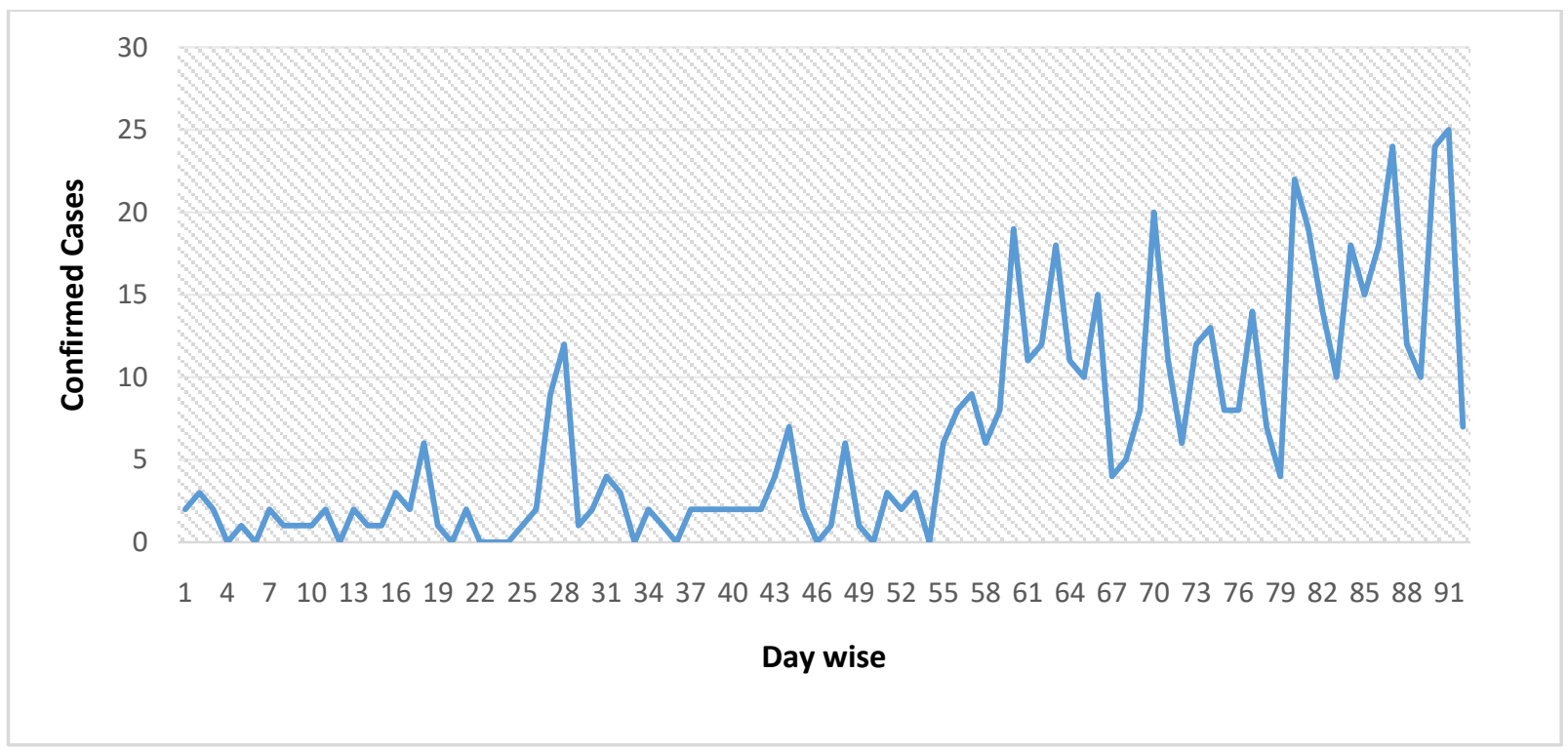

Fig-4: Self-similarity behavior of COVID-19 Confirmed cases

From Fig-3 and Fig-4,we observed that there was a self-similar pattern of the number of patients arrivals to the COVID-19 test. And even COVID-19 confirmed cases also resemble self-similarity. These findings warrant further inquiry. It showed apparent survival of self-similarity in the patient's arrival and their confirmation of positive cases. 


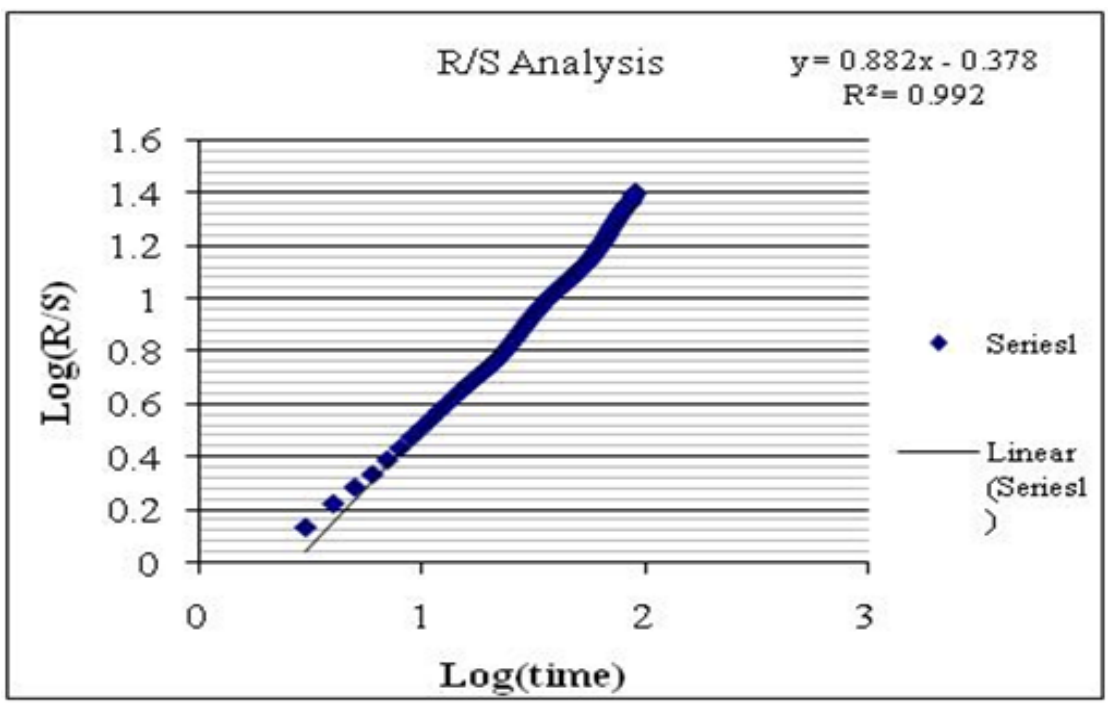

Fig-5 Hurst Index Parameter by R/S Method

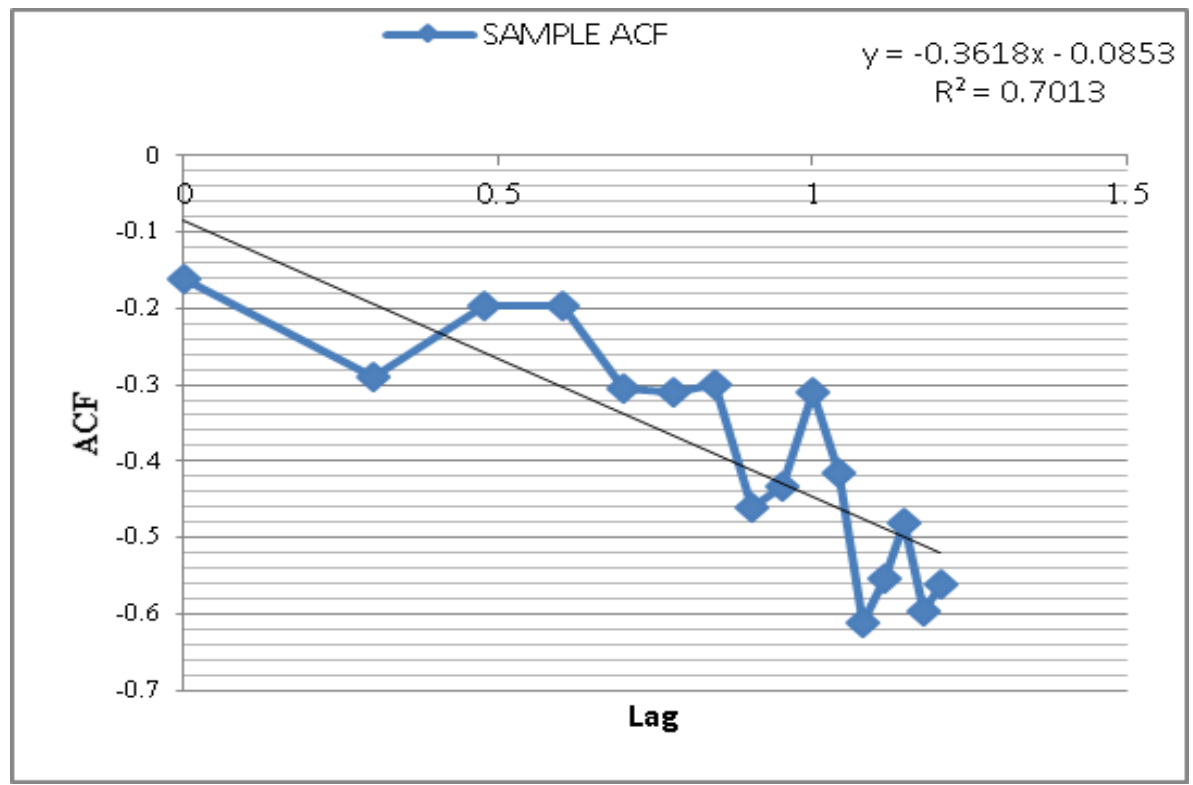

Fig-6 Hurst Index Parameter by Correlogram Method

Calculating queue length $(\bar{L}): \quad \bar{L}=\frac{\rho^{0.5 /(1-H)}}{(1-\rho)^{H /(1-H)}}$

Traffic intensity versus Queue Length using M/M/1 model shows that increase in queue traffic intensity also increases. By Hurst Index parameter by R/S Method is 0.8824 , and corresponding queue length is $\bar{L}$ is calculated in Table-5, and by using the Correlogram method is 0.8195 . For this, we computed queue length is $\bar{L}$ in the same Traffic intensity versus $\bar{L}$ using Hurst index parameter showed inFig- 8 . As shown in the figures, we can conclude that as traffic intensity increases, the queues average length increases, which is expected. As well, when $H$ increases, the average size of the queue increases resulted in Table-5. This result concurs with our observation. 


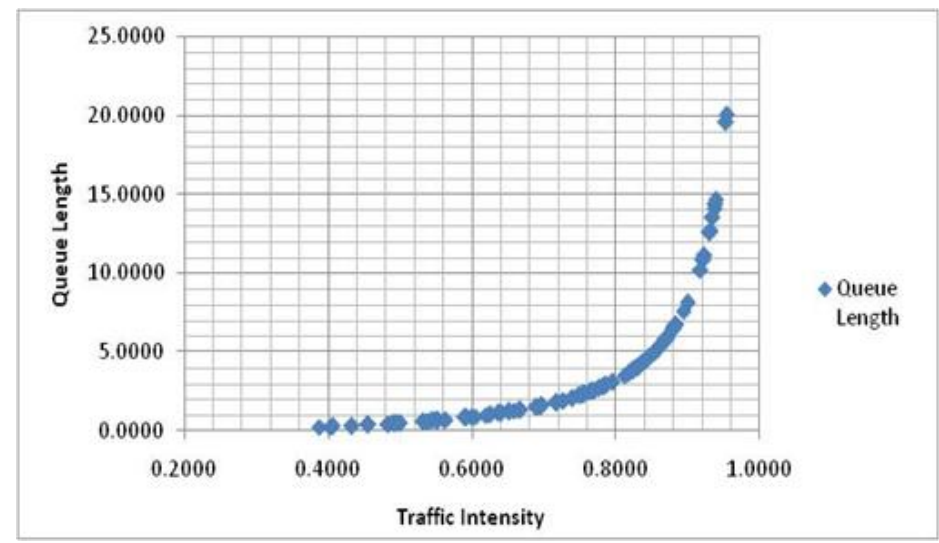

Fig-7 Queue length V/s Traffic intensity of COVID-19 patients M/M/1 model

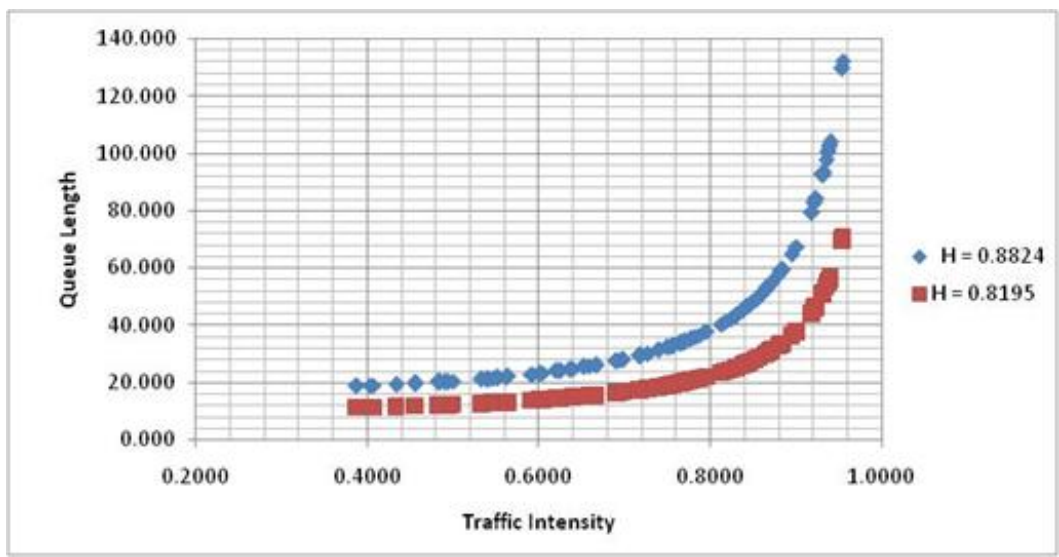

Fig-8 Queue length V/s Traffic intensity of COVID-19 patients' arrival

\section{Conclusion:}

In this paper's real information related to Covid-19 data from the healthcare, the center shows self-similarity using Hurst index methods. Also, calculate the average queue length using $\mathrm{M} / \mathrm{M} / 1$ formulae and using the mean size, and we compare results with $\mathrm{M} / \mathrm{M} / 1$ model and queue length using the Hurst index formula. Based on the concept of self-similarity and finding the intensity of traffic using $\mathrm{H}$ is easy to measure the queue length and as the $\mathrm{H}$ value increases average queue length also increases This analysis is useful to design the Healthcare centers based on the result of the traffic intensity improve service facilities of the centers in local areas.

\section{Acknowledgement:}

I sincerely thank Dr.Raghunath and his staff members of Dipali Hospital, Hosapete, Ballari(Dist) who supported me to collect data of covid-19 samples testing information in this pandemic period.

I sincerely thank patients who given their personal details and supporting for my research work.

\section{References:}

[1] Erlang, A.K., The theory of probabilities and telephone conversations, TidsskriftMathematica, Vol. 20, pp. 33-39,(1909).

[2] Ali, I., and Omar M L A., 2020. COVID-19: Disease, management, treatment, and social impact. The science of the total environment, 728(2020): 138861.

[3] Banerjee, A., Kulcsar, K., Misra, V., Frieman, M., Mossman, K., 2019. Bats and 
coronaviruses. Viruses. 11(1): E41.

[4] Cochran, J.K., and Roche, K.T., 2009. A multi-class queuing network analysis methodology

for improving hospital emergency department performance. Computers Operations Research,36:1497-512.

[5] Gao, W.J., Zheng, K., Ke, J., and Li, L.M., 2020. Advances on the asymptomatic infection of COVID-19. Chinese Journal of Epidemiology, 41.

[6] Meng, Q., Khoo, H.L. (2009). Self-similar characteristics of vehicle arrival pattern on highways. Journal of Transportation Engineering 135 (11): 864-872.ScholarBank@NUS Repository. https://doi.org/10.1061/(ASCE)0733-947X(2009)135:11(864)

[7] Guo, Y., Cao, Q., and Hong, Z., 2020. The origin, transmission, and clinical therapies on coronavirus disease 2019 (COVID-19) outbreak - an update on the status. Military Medical

[8] Guo, Y., Wang, J., Yue, X., He, S., and Zhang, X., 2010. The Optimization Model of Hospital Sick Beds' Rational Arrangements. In International Conference on Information Computing and Applications, 40-47, Springer, Berlin, Heidelberg.

[9] Harapan, H., Itoh, N., Yufika, A., Winardi, W., Kim, S., Te, H., Megawati, D., Hayati, Z.,Wagner, A.L. and Mudatsir, M., 2020. Coronavirus disease 2019 (COVID-19): A literature review. Journal of Infection and Public Health, 13(5), 667-673

[10]Hu, Z., Song, C., Xu, C., Jin, G., Chen, Y., and Xu, X., 2020. Clinical characteristics of 24 asymptomatic infections with COVID-19 screened among close contacts in Nanjing,China. Science China Life Science, 63(5):706-711.

[11]Gospodinov, M., E. Gospodinova. "Generator of fractional Gaussian noise for modeling self-similar network traffic," CompSysTech'2005.

[12] Brockwell, J. P., \& Davis, A. R. (1996). Introduction to Time Series and Forecasting, Springer-Verlag New York Inc.

[13] Beran, J., Taqqu, M.S. and Willinger, W., Long-range dependence in variable bit rate traffic, IEEE Trans. on Communications, Vol. 43, pp. 1566-1579, (1995)

[14] D.Mallikarjuna Reddy, A.M Girija, and PushpalathaSarla. "An Application of Queuing System to patient satisfaction at a selected hospital-A field Study" AIP Conference Proceedings 2246, 020111(2020), https://doi.org/10.1063/5.0014439.

[15] Nosek Jr, R. A., and Wilson, J. P., 2001. Queuing theory and customer satisfaction: A Review of terminology, trends, and applications to pharmacy practice. Hospital pharmacy, 36(3),275-279.

[16] PushpalathaSarla, Mallikarjuna Reddy "Analytical Study of Self-similar Type Traffic Data-Queuing Techniques," AIP Conference Proceedings 2246, 020006(2020), https://doi.org/10.1063/5.0014432

[17] WHO, 2020. Coronavirus disease 2019 (COVID-19) https://www.who.int/emergencies/diseases/novel-coronavirus-2019/situation-reports Situation Report-100.

[18] Zhu, N., Zhang, D., Wang, W., Li, X., Yang, B., and Song, J. 2020. A novel coronavirus from patients with pneumonia in China. The New England Journal of Medicine, 382: 727-733.

[19] http://www.iaeme.com/IJARET/index.asp 23 editor@iaeme.com.

[20] Hurst H 2005 Hurst parameter of self-similar network traffic International Conference on Computer Systems and Tech

[21] Roughness 2003 Length Method for Estimation Hurst Exponent and Fractal Dimension of Traces Help Benoit 1.3 version SoftwareTruSoft International Inc.

[22] M.Gospodinov and E. Gospodinova, "The graphical methods for estimating Hurst parameter of self-similar network traffic," in proceedings of ICCST, 2005.

[23] Himanshu Mittal and Naresh Sharmaonline "A probabilistic model for The Assessment of Queuing time of Coronavirusdisease(COVID-19) Patients using Queuing Model” ISSN Print: 0976-6480 http://www.iaeme.com/IJARET/issues.asp?JType=IJARET\&VType=11\&IType=8. 